\title{
Hepatic disease as the first manifestation of progressive myoclonus epilepsy of Lafora
}

P. Gómez-Garre, PhD*

E. Gutiérrez-Delicado, MD*

C. Gómez-Abad, PhD

J. Morales-Corraliza, $\mathrm{PhD}$

V.E. Villanueva, MD

S. Rodríguez de Córdoba, PhD

J. Larrauri, MD, PhD

M. Gutiérrez, MD, PhD

J. Berciano, MD, PhD

J.M. Serratosa, MD, $\mathrm{PhD}$

Address correspondence and reprint requests to Dr. José M Serratosa, Servicio de

Neurología, Fundación Jiménez Díaz, Avda Reyes Católicos, 2, 28040 Madrid, Spain serratosa@telefonica.net
ABSTRACT Background: Lafora disease (LD; progressive myoclonus epilepsy type 2; EPM2) is an autosomal recessive disorder caused by mutations in the EPM2A and EPM2B genes. LD is characterized by the presence of strongly PAS-positive intracellular inclusions (Lafora bodies) in several tissues. Glycogen storage disease type IV (GSD-IV; Andersen disease) is an autosomal recessive disorder characterized by cirrhosis leading to severe liver failure. GSD-IV has been associated with mutations in the glycogen branching enzyme gene (GBE). Histopathologic changes of the liver in both diseases show an identical appearance, although cirrhosis has never been described in patients with LD. We report a LD family in which the proband presented severe liver failure at onset of the disease. Methods: Clinical histories, physical and neurologic examination, laboratory tests, EEGs, MRI of the brain, and liver or axillary skin biopsies were performed in the two affected siblings. The diagnosis was confirmed by molecular genetic analysis of the EPM2A, EPM2B, and GBE genes and loci. Results: During the first decade of life, abnormalities in liver function tests were detected in the two affected siblings. The proband's liver dysfunction was severe enough to require liver transplantation. Subsequently, both sibs developed LD. Mutation analysis of EPM2A revealed a homozygous Arg241stop mutation in both patients. Conclusions: This is the first description of severe hepatic dysfunction as the initial clinical manifestation of $L D$. The phenotypic differences between the two affected siblings suggest that modifier genes must condition clinical expression of the disease outside the CNS. NEUROLOGY 2007;68:1369-1373

Accumulation of polyglucosans represents the morphologic manifestation of several clinically heterogeneous disorders such as Lafora disease, glycogen storage disease type IV, adult polyglucosan body disease, and some cases of phosphofructokinase deficiency (Tauri disease).

Lafora disease or progressive myoclonus epilepsy of the Lafora type (EPM2 [MIN254780]) is an autosomal recessive form of progressive myoclonus epilepsy (PME) that initially manifests during adolescence and is characterized by epilepsy, myoclonus, and progressive neurologic deterioration. The diagnosis is sustained by the presence of periodic acid-Schiff (PAS) positive glycogen-like intracellular inclusion bodies (Lafora bodies). Lafora bodies, first described by Gonzalo R. Lafora in 1911, consist of an abnormal glucose polymer. ${ }^{1,2}$ Lafora originally called the inclusion bodies "intracellular amyloid bodies" when he observed them in the brain and spinal cord of an adolescent patient who presented a progressive and fatal form of myoclonic epilepsy. In 1955, Harriman and Millar showed that the inclusions were not limited to the CNS and described similar intracellular inclusions in the heart and liver of one patient with Lafora disease. ${ }^{3}$ Lafora disease initially manifests during adolescence, the most common age at onset being between 10 and 17 years. Generalized tonic-clonic seizures, absences, drop attacks, or partial visual seizures are usually the first manifestation, followed soon after by asymmetric as well as massive myoclonic jerks. As the disease progresses, the myoclonus increases in frequency and becomes constant. A rapidly progressive dementia

\footnotetext{
*These authors contributed equally to this work.

From Laboratorio y Servicio de Neurología (P.G.-G., E.G.-D., C.G.-A., J.M.-C., V.E.V., J.M.S.), Fundación Jiménez Díaz, Madrid; Centro de Investigaciones Biológicas (S.R.C.), Consejo Superior de Investigaciones Científicas, Madrid; Departamento de Anatomía Patológica (J.L., M.G.), Hospital Universitario La Paz, Madrid; and Departamento de Neurología (J.B.), Hospital Universitario Marqués de Valdecilla (UC), Santander, Spain. V.E.V. is currently affiliated with Unidad de Epilepsia, Servicio de Neurología, Hospital La Fe, Valencia, Spain.

Supported by the Spanish Ministry of Science and Education (SAF2004/07151) and Fondo de Investigaciones Sanitarias (PI020536 and C03/06).

Disclosure: The authors report no conflicts of interest.
} 
(A) Spanish Lafora disease family. Black symbols indicate affected individuals. (B) Single-strand conformational

polymorphism analysis. Individuals LD122-1, LD122-2, and LD122-3 are denoted as 1, 2, and 3 . Individual C1 is an unaffected control. Individual C2 is affected with the Arg241stop mutation in homozygosis. Individuals 1 and 2 carry the mutation in heterozygosis and Individual 3 in homozygosis. (C) DNA sequence of exon 4 of the EPM2A gene showing the $\mathrm{C} \rightarrow$ T change resulting in the replacement of arginine 241 by a stop codon.
Figure 1 Spanish Lafora disease family

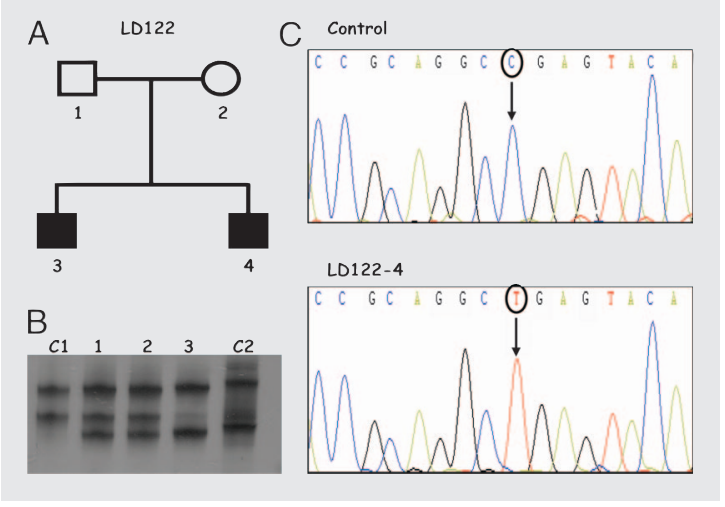

with apraxia, aphasia, and visual loss ensues, leading patients to a vegetative stage and death, usually within less than a decade of first symptoms. Two genes have been associated with Lafora disease: EPM2A (chromosome 6q24) and EPM2B (chromosome 6p22.3). EPM2A encodes a protein tyrosine phosphatase (LAFPTPase or laforin). ${ }^{4,5}$ In spite of the remarkable allelic heterogeneity in the EPM2A gene, the R241stop mutation has been found in approximately $40 \%$ of Lafora disease patients with mutations in this gene. ${ }^{6,7}$ The EPM2B gene (also called NHLRC1) encodes an E3 ubiquitin ligase (malin) and the most frequent mutation found is P69A. ${ }^{8}$

Glycogen storage disease or glycogenosis type IV, also known as amylopectinosis or Andersen disease (MIN23250), is an autosomal recessive disorder caused by a deficiency of glycogen-branching enzyme (GBE) activity due to mutations in the GBE1 gene. ${ }^{9} \mathrm{~A}$ diagnosis can be made on the basis of the study of branching enzyme activity in erythrocytes. ${ }^{10}$ Glycogenosis type IV is highly heterogeneous in terms of tissue involvement, clinical manifestations, and age at onset. The classic form of glycogenosis type IV is characterized by rapidly progressive hepatosplenomegaly and liver failure leading to either liver transplantation or death by the age of 5 years. ${ }^{9}$ In addition to the classic form, variants such as a milder nonprogressive hepatic disease, ${ }^{11}$ neuromuscular forms varying in onset and severity (from a fatal neonatal disease ${ }^{12}$ to a mild adult myopathy ${ }^{13}$ ), a cardiopathic form of childhood, ${ }^{14}$ or a variant with multisystem involvement including liver and muscle ${ }^{15}$ have been reported. GBE belongs to the alpha-amylase family and is required for glycogen synthesis. It catalyzes the formation of alpha 1,6glucosidic branches that play an important role increasing the solubility of the molecule and is most highly expressed in liver and muscle. Typically, hepatocytes in glycogenosis type IV contain glycogen PAS-positive inclusions resulting in a ground glass appearance. This stored glycogen shows fewer branch points and longer outer chains resembling amylopectin. For this reason the disease is also referred to as amylopectinosis. Ultrastructurally, the inclusion bodies of Lafora disease and glycogenosis type IV appear quite similar. ${ }^{16}$ Liver fibrosis, which can progress to cirrhosis, is a frequent finding in glycogenosis type IV. However, only mild to moderate periportal fibrosis has been described in the liver of patients with Lafora disease. ${ }^{17}$

Here, we present a clinical and molecular genetic study of two siblings with Lafora disease, one of whom developed a progressive liver cirrhosis and failure, requiring liver transplantation.

METHODS Patients and samples. We studied two siblings from a Spanish family (figure 1A). Both presented epilepsy, myoclonus, rapidly progressive neurologic deterioration, and a slow background activity with polyspike-wave complexes in the EEG..$^{18,19}$ A biopsy of skin from the proband and liver biopsy from the younger brother showed the characteristic PAS-positive Lafora bodies in both. Blood was collected from patients and their relatives after informed consent.

DNA samples were obtained from peripheral blood lymphocytes using standard methods. The study was approved by the Ethics Committee of the Fundación Jiménez Díaz.

Microsatellite analysis. Analysis of microsatellite polymorphisms on chromosomes 6q24 and 3p12 was performed by PCR using total human genomic DNA. Amplification was performed in a total volume of $10 \mu \mathrm{L}$ containing $40 \mathrm{ng}$ of genomic DNA and isotopic phosphate. Samples were resolved on $6.5 \%$ polyacrylamide sequencing gels and bands were visualized using Kodak XAR films for 1 to 14 hours.

Single-strand conformational polymorphism (SSCP) analysis. SSCP analysis ${ }^{20}$ was performed by PCR, using total genomic DNA, using the GenePhor DNA Electrophoresis System (Amersham Pharmacia Biotech). Amplification was performed in a total volume of $10 \mu \mathrm{L}$ containing $60 \mathrm{ng}$ of genomic DNA. Samples were resolved on $12.5 \%$ non-denaturing polyacrylamide gels with the GeneGel Excel 12.5/24 kit (Amersham 
(A) Histologic study of the liver of Individual LD112-3 showing loss of hepatic structure and confluent bands of collagen forming nodules (Masson's trichromic stain). (B) Ground-glass appearance of hepatocytes as expression of the Lafora bodies (arrow)

Figure 2 Histologic study of the liver of Individual LD112-3

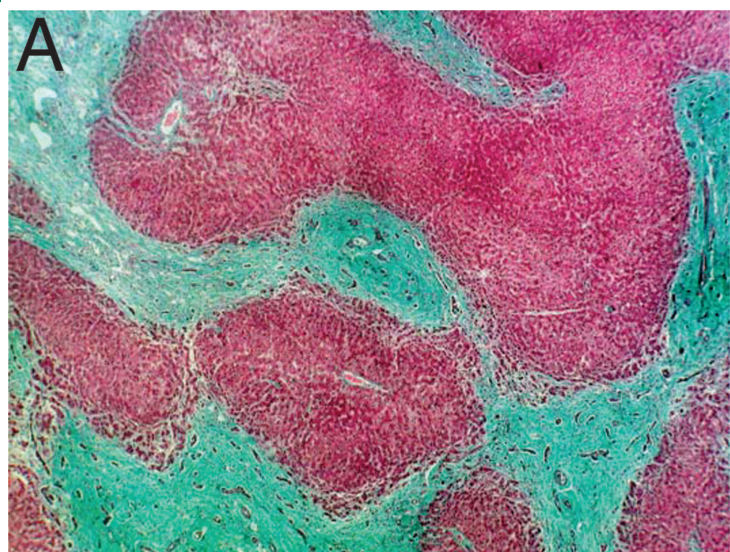

$10 \mathrm{X}$

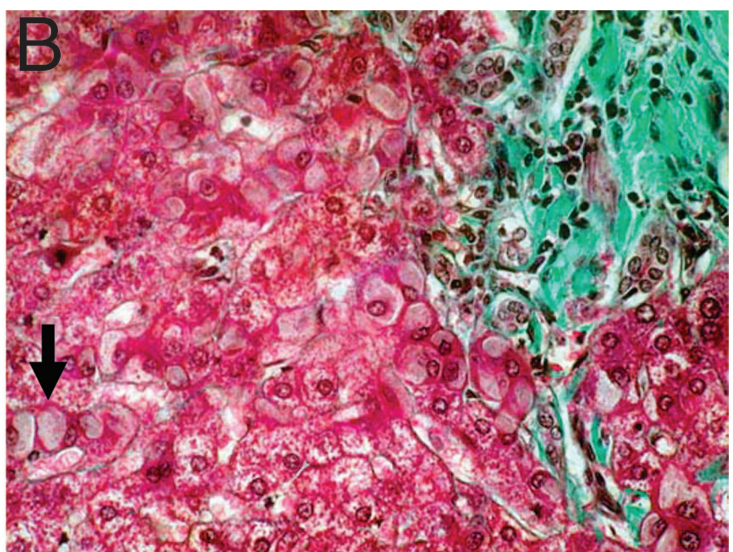

$40 x$
Pharmacia Biotech) and silver stained using the PlusOne DNA Silver Staining Kit (Amersham Pharmacia Biotech).

Sequencing of exons. Exons 1, 2, 3, and 4 of the EPM2A gene, ${ }^{6}$ exon 1 of the EPM2B gene, ${ }^{8}$ and exons 1 to 16 of the $G B E$ gene were amplified from genomic DNA with specific primers using standard methods. The corresponding PCR products were purified by agarose gel electrophoresis and extracted with the Qiaquick Gel Extraction Kit (Qiagen). Direct sequencing of PCR products was performed with a dye-terminator cyclesequencing kit (Perkin-Elmer) using Taq FS DNA polymerase. Sequences were resolved on an ABI PRISM 377 automatic sequencer, and the results analyzed with the ABI Analysis software (version 3.1).

RESULTS Clinical findings. Case 1. The proband presented growth retardation with height and weight deficiencies since early infancy. At age 7, laboratory tests revealed an increase in hepatic transaminase levels (alanine aminotransferase 321, aspartate aminotransferase 380) and abdominal distension, ascites, and increasing splenomegaly due to portal hypertension appeared. Hemorrhagic complications occurred subsequently and a severe hepatic failure was detected. A liver biopsy was performed at age 9. The histopathologic study showed ground-glass appearance of hepatocytes demonstrating positive Best carmine staining and lack of staining with orcein. Severe periportal stellate fibrosis was observed suggesting type IV glycogen storage disease (figure $2, \mathrm{~A}$ and $\mathrm{B}$ ). He required liver transplantation at age 10 . No clinical complications occurred during follow-up.

At age 16 he noticed transient light spots during a school test. He had his first generalized tonic-clonic seizure the following day. Six months later, myoclonic jerks were noticed. At first, these were asymmetric, segmental myoclonic jerks and occurred occasionally. During the following year, generalized tonic-clonic seizures increased in frequency and massive myoclonus appeared. Seizures were refrac- tory to antiepileptic drugs. Progressive intellectual impairment occurred during the next 2 years.

An EEG showed diffuse slow background activity with bilateral paroxysms of spike-wave and polyspike-wave complexes. Marked photosensitivity was noted. Brain magnetic resonance showed cerebral atrophy and typical Lafora bodies were seen in an axillary skin biopsy.

Case 2. The proband's younger brother presented an increase of liver transaminases levels in a routine analysis at age 6 (alanine aminotransferase 112, aspartate aminotransferase 80). Although he remained clinically asymptomatic, a liver biopsy was performed at age 9. Liver biopsy revealed PASpositive intracellular inclusion bodies with normal lobular architecture. No periportal fibrosis was observed. At age 14 he initially noticed occasional episodes consisting of seeing sudden and transient brilliant spots. These episodes lasted for few seconds and were especially frequent when he was watching television. Massive myoclonic jerks appeared 3 months later and he had his first generalized tonic-clonic seizure 1 year after the onset of neurologic symptoms. EEG showed a slow background activity, occipital spikes increasing with intermittent photic stimulation, and paroxysms of irregular spike-wave activity on both frontotemporal regions. During follow-up, myoclonic epilepsy and neurologic deterioration progressively developed but no hepatic dysfunction was detected.

Genetic findings. SSCP and mutational analysis of exons 1, 2, 3, and 4 of the EPM2A gene revealed a homozygous Arg241stop mutation in both siblings (figure 1, B and C), confirming the diagnosis of Lafora disease. Exons 1 to 16 of the GBE gene and exon 1 of EPM2B gene were also analyzed in both siblings in order to search for mutations. No mutations were found. 
Haplotype analysis of the EPM2A and GBE genes was also performed and showed that both relatives presented identical haplotype for these loci (data not shown).

DISCUSSION Lafora disease has been considered a clinically homogeneous degenerative disorder manifested by progressive myoclonic epilepsy. Atypical Lafora disease with an early onset cognitive deficit phenotype has been recently reported by different authors in a minority of patients. ${ }^{21-23}$ However, in no case of Lafora disease caused by mutations in $E P M 2 A$ or EPM2B genes have initial clinical symptoms outside the CNS been reported. Here we describe the first Lafora disease family in which the proband presented a severe liver failure resembling glycogenosis type IV at onset of the disease. His younger brother showed abnormal liver function tests in a routine analysis although he was clinically asymptomatic and no signs of hepatic failure were detected during his follow-up. Both siblings subsequently developed the typical picture of progressive myoclonus epilepsy of Lafora type. PAS-positive inclusions were observed in an axillary skin biopsy from the proband and in a liver biopsy from his younger brother. The diagnosis of Lafora disease was confirmed by mutation analysis of the EPM2A gene, which revealed an Arg241stop mutation in homozygosis in both siblings. Interestingly, only the proband presented severe liver failure preceding the onset of progressive myoclonus epilepsy.

Histopathologic study of the liver in the proband showed ground-glass appearance of hepatocytes with PAS-positive inclusions and cirrhosis, leading to the diagnosis of glycogenosis type IV. Histopathologic changes of the liver in Lafora disease and glycogenosis type IV show an identical appearance. The similarity applies especially to the ground glass hepatocytes containing PAS-positive inclusions. Histochemical reactions of stored material are similar. However, patients with glycogenosis type IV typically develop cirrhosis while only mild to moderate periportal fibrosis has been described in patients with Lafora disease. ${ }^{17}$ The clinical picture and the histopathologic findings in the proband strongly suggested the diagnosis of glycogenosis type IV. Mutation analysis of the complete coding region of the GBE gene did not reveal any mutations and haplotype analysis of the chromosomal region containing the GBE gene revealed identical haplotypes for both sibs, suggesting that this gene is not responsible for the liver failure present in the proband.

Similarities between Lafora and glycogenosis type IV diseases suggest that these two conditions are closely related and may share a common metabolic disturbance conditioning failures at different levels of the same metabolic pathway. Concomitant enzyme deficiencies in the same individual causing an unusual glycogenosis with extensive neuronal polyglucosan storage has been reported. ${ }^{24}$ This is an extremely rare condition but it is reasonable to think that a similar situation may account for the unusual clinical findings found in the patient here presented. This patient presents a combination of two different clinical phenotypes included in the group of glycogen metabolic pathway disorders. A homozygous mutation in EPM $2 A$ and no mutation in GBE were found. The most plausible hypothesis is that other unidentified modifier genes or genes involved in glycogen metabolism might condition the clinical expression of Lafora disease outside the CNS.

\section{ACKNOWLEDGMENT}

The authors thank family members who participated in this study.

Received August 21, 2006. Accepted in final form December 15, 2006.

\section{REFERENCES}

1. Lafora GR. Über das vorkommen amyloider körperchen im inner der ganglienzellen; zugleich ein zum studium der amyloiden substanz im nervensystem. Virchows Arch [Pathol Anat] 1911;205:295-303.

2. Lafora GR, Glueck B. Beitrag zur histopathologie der myoklonischen epilepsie. Z Gesamte Neurol Psychiatr 1911;6:1-14.

3. Harriman DG, Millar JH. Progressive familial myoclonic epilepsy in three families: its clinical features and pathological basis. Brain 1955;78:325-349.

4. Minassian BA, Lee JR, Herbrick JA, et al. Mutations in a gene encoding a novel protein tyrosine phosphatase cause progressive myoclonus epilepsy. Nat Genet 1998;20:171174.

5. Serratosa JM, Gomez-Garre P, Gallardo ME, et al. A novel protein tyrosine phosphatase gene is mutated in progressive myoclonus epilepsy of the Lafora type (EPM2). Hum Mol Genet 1999;8:345-352.

6. Gomez-Garre P, Sanz Y, Rodriguez De Cordoba SR, Serratosa JM. Mutational spectrum of the EPM2A gene in progressive myoclonus epilepsy of Lafora: high degree of allelic heterogeneity and prevalence of deletions. Eur J Hum Genet 2000;8:946-954.

7. Ki CS, Kong SY, Seo DW, Hong SB, Kim HJ, Kim JW. Two novel mutations in the EPM2A gene in a Korean patient with Lafora's progressive myoclonus epilepsy. J Hum Genet 2003;48:51-54.

8. Chan EM, Young EJ, Ianzano L, et al. Mutations in NHLRC1 cause progressive myoclonus epilepsy. Nat Genet 2003;35:125-127.

9. Bao Y, Kishnani P, Wu JY, Chen VT. Hepatic and neuromuscular forms of glycogen storage disease type IV caused by mutations in the same glycogen-branching enzyme gene. J Clin Invest 1996;97:941-948.

10. Shin YS, Steiguber H, Klemm P, Endres W, Schwab O, Wolff G. Branching enzyme in erythrocytes: detection of 
type IV glycogenosis homozygotes and heterozygotes. J Inherit Metab Dis 1988;11(suppl 2):252-254.

11. Greene HL, Brown BI, McClenathan DT, Agostini RM Jr, Taylor SR. A new variant of type IV glycogenosis: deficiency of branching enzyme activity without apparent progressive liver disease. Hepatology 1988;8:302.

12. Van Noort G, Straks W, Van Diggelen OP, Hennekam RC. A congenital variant of glycogenosis type IV. Pediatr Pathol 1993;13:685-698.

13. Ferguson IT, Mahon M, Cumming WJ. An adult case of Andersen's disease-Type IV glycogenosis. A clinical, histochemical, ultrastructural and biochemical study. J Neurol Sci 1983;60:337-351.

14. Servidei S, Riepe RE, Langston C, et al. Severe cardiopathy in branching enzyme deficiency. J Pediatr 1987;111: $51-56$.

15. Bruno C, DiRocco M, Lamba LD, et al. A novel missense mutation in the glycogen branching enzyme gene in a child with myopathy and hepatopathy. Neuromuscul Disord 1999;9:403-407.

16. Ishihara T, Yokota T, Yamashita Y, et al. Comparative study of the intracytoplasmic inclusions in Lafora disease and type IV glycogenosis by electron microscopy. Acta Pathol Jpn 1987;37:1591-1601.

17. Schwarz GA, Yanoff M. Lafora's disease: distinct clinicopathologic form of Unverricht's syndrome. Arch Neurol 1965;12:172-188.
18. Tassinari CA, Bureau-Paillas M, Dalla Bernardina B, et al. La maladie de Lafora. Rev EEG Neurophysiol Clin 1978;8:107-122.

19. Van Heycop Ten Ham MV. Lafora disease, a form of progressive myoclonus epilepsy. In: Vinken PJ, Bruin GW, eds. Handbook of clinical neurology. North Holland: Amsterdam, 1974;15:382-422.

20. Orita M, Iwahana H, Kanazawa H, Hayashi K, Sekiya T. Detection of polymorphisms of human DNA by gel electrophoresis as single-strand conformation polymorphism. Proc Natl Acad Sci USA 1989;86:2766-2770.

21. Ganesh S, Delgado-Escueta AV, Suzuki T, et al. Genotype-phenotype correlations for EPM2A mutations in Lafora's progressive myoclonus epilepsy: exon 1 mutations associate with an early-onset cognitive deficit subphenotype. Hum Mol Genet 2002;11:1263-1271.

22. Annesi G, Sofia V, Gambardella A, et al. A novel exon 1 mutation in a patient with atypical Lafora progressive myoclonus epilepsy seen as childhood-onset cognitive deficit. Epilepsia 2004;45:294-295.

23. Gómez-Abad C, Gómez-Garre P, Gutiérrez-Delicado E, et al. Lafora disease due to EPM2B mutations. A clinical and genetic study. Neurology 2005;64:982-986.

24. Herrick MK, Twiss JL, Vladutiu GD, Glasscock GF, Horoupian DS. Concomitant branching enzyme and phosphorylase deficiencies. An unusual glycogenosis with extensive neuronal polyglucosan storage. J Neuropathol Exp Neurol 1994;53:239-246.

\section{Resident and Fellow Section: Call for Teaching Videos}

The Neurology Resident section is featured online at www.neurology.org. The Editorial Team of this section is seeking teaching videos that will illustrate classic or uncommon findings on movement disorders. Such videos will aid in the recognition of such disorders. Instructions for formatting videos can be found in the Information for Authors at www.neurology.org. 